\title{
Trombosis protésica: la ecocardiografía nuestra gran aliada
}

\author{
Joffrey Eduardo Luján Valencia* \\ Carmen Riaza Ocio** \\ Fausto Librada Escribano*** \\ Lucía Álvarez Lacruz*** \\ Pedro María Azcárate Agüero****
}

Correspondencia

Joffrey Eduardo Luján Valencia

edu_luv@hotmail.com

* Departamento de Cardiología. Hospital Marqués de Valdecilla. Santander (Cantabria). España

** Departamento de Medicina Familiar y Comunitaria. Centro de Salud Gonzalo de Berceo. Logroño (La Rioja). España

*** Departamento de Cardiología. Hospital San Pedro. Logroño (La Rioja). España

Recibido: $27 / 08 / 2020$

Aceptado: 08/10/2020

En línea: 31/12/2020

Citar como: Luján Valencia JE, Riaza Ocio C, Librada Escribano F, Álvarez Lacruz L, Azcárate Agüero PM. Trombosis protésica: la ecocardiografía nuestra gran aliada. Rev Ecocar Pract (RETIC). 2020 (Dic); 3 (3): 26-29. doi: 10.37615/retic.v3n3a11.

Cite this as: Luján Valencia JE, Riaza Ocio C, Librada Escribano F, Álvarez Lacruz L, Azcárate Agüero PM. Prosthetic thrombosis: echocardiography our great ally. Rev Ecocar Pract (RETIC). 2020 (Dic); 3 (3): 26-29. doi: 10.37615/retic.v3n3a11.

\author{
Palabras clave \\ $\triangleright$ Implantación de prótesis \\ valvulares cardíacas \\ $\triangleright$ Trombosis \\ $\triangleright$ Fibrinólisis
}

\begin{abstract}
RESUMEN
Las prótesis valvulares constituyen el tratamiento definitivo de muchas valvulopatías avanzadas. Sin embargo, no están exentas de complicaciones como la endocarditis infecciosa, la obstrucción de la válvula protésica (trombosis y pannus), eventos embólicos o insuficiencias periprotésicas. La trombosis de una prótesis valvular debería ser sospechada en cualquier paciente con disnea o fenómeno embólico reciente. Un apropiado diagnóstico resulta fundamental para la elección de la estrategia terapéutica. Ésta implica desde la intensificación de anticoagulación a medidas más agresivas como la cirugía de emergencia, la fibrinólisis e incluso medidas paliativas. Nuestro objetivo es resaltar el papel de la ecocardiografía en cada fase de su manejo.
\end{abstract}

ABSTRACT
Valve prostheses are the final treatment for many advanced valvular diseases. However, they are not exempt from
complications. For example, infective endocarditis, obstruction of the prosthetic valve (thrombosis and pannus),
embolic events and periprosthetic regurgitation. Prosthetic valve thrombosis should be suspected in any patient
with dyspnea or embolic phenomenon of recent onset. An appropriate diagnosis is essential in order to choose
the best therapeutic strategy. This implies from the intensification of anticoagulation to more aggressive measures
such as emergency surgery, fibrinolysis and even palliative measures. Our aim is to highlight the role of echocar-
diography in each phase of the management of this complication.

\section{Presentación del caso}

Mujer de 73 años con antecedentes de dislipidemia, hipertensión arterial, portadora de prótesis mecánicas en posición aórtica y mitral desde hacía 2 años por valvulopatía degenerativa y con hemicolectomía izquierda por adenocarcinoma de colon intervenida un mes antes.

Acudía a Urgencias por disnea progresiva, ortopnea y edemas de 4 días de evolución. A la exploración, mal estado general, consciente, tensión arterial $100 / 55 \mathrm{mmHg}$, frecuencia cardíaca $100 \mathrm{lpm}, \mathrm{SatO}_{2} 88 \%$ con tiraje intercostal. Auscultación rítmica, con soplo sistólico en foco mitral y ausencia de clic metálicos, crepitantes bilaterales hasta vértices pulmonares; ingurgitación yugular y edemas en miembros inferiores. En la analítica destacaba deterioro de función renal ( $\mathrm{Cr} 1,3 \mathrm{mg} / \mathrm{d}$ ), elevación de marcadores cardíacos (proBNP $7.267 \mathrm{pg} / \mathrm{ml}$ y troponina T ultrasensible 68,1 ng/l), INR 1,17 y hemograma normal. Electrocardiograma en ritmo sinusal a 95 Ipm y radiografía de tórax compatible con edema agudo de pulmón (Figura 1). Presentaba niveles infraterapéuticos de anticoagulación debido a la terapia puente con heparinas de bajo peso molecular (HBPM) como consecuencia de la intervención quirúrgica en semanas previas.

Se realizó ecocardiografía transtorácica (ETT) en la que se constataron datos indirectos de disfunción protésica: aumento de gradientes con respecto a ecocardiogramas previos (gradiente mitral basal medio previo de $4 \mathrm{mmHg}$ que ahora se elevaba a $25 \mathrm{mmHg}$, y gradiente aórtico basal medio previo de 10 $\mathrm{mmHg}$ que ahora se elevaba a $35 \mathrm{mmHg}$ ) (Vídeo 1 y Figura 2). La paciente presentaba, además, aumento de la presión arterial pulmonar (Figura 3).

La situación de edema agudo de pulmón y la alta sospecha clínica de trombosis valvular hacían de la cirugía la opción más adecuada, sin embargo, dado el 
elevado riesgo quirúrgico, se decidió tratamiento depletivo y posterior inicio de protocolo de fibrinólisis lenta con alteplasa en 24 horas, previa información de los riesgos de dicho procedimiento. Tras dos ciclos de fibrinólisis lenta, se realizó ecocardiografía transesofágica (ETE), apreciándose casi la normalización de los gradientes (gradiente mitral medio de 4,5 mmHg y aórtico medio de 12 $\mathrm{mmHg}$ ), aunque persistía imagen compatible con trombos de menor tamaño que interferían con la apertura y cierre del disco posterior de prótesis mitral, decidiéndose continuar con heparina sódica y añadiendo ácido acetilsalicílico (AAS) (Figura 4; Vídeo 2 y Vídeo 3).

Tras 7 días, se realizó ETT, comprobándose la progresión del tamaño del trombo (Vídeo 4 y Vídeo 5) y el empeoramiento de gradientes transvalvulares: mitral medio $6 \mathrm{mmHg}$ y aórtico de $19 \mathrm{mmHg}$ (Figura 5). Ello nos llevó a administrar un tercer ciclo de fibrinólisis que permitió la normalización del gradiente mitral a 4 mmHg y aórtico a $15 \mathrm{mmHg}$.

Una vez resuelta la obstrucción valvular, y comprobándose la ausencia de trombos de tamaño significativo mediante ETE (móvil $>5 \mathrm{~mm}$ o cualquier trombo $>1 \mathrm{~cm}^{2}$ ), se decidió alta con seguimiento ambulatorio a los 3 meses favorable.

\section{Estudio por imagen}

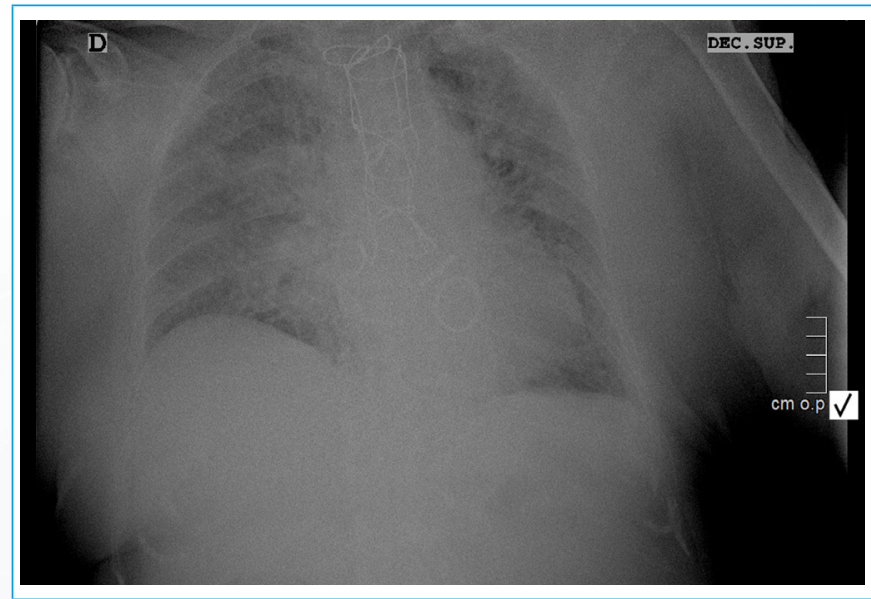

Figura 1. Radiografía de tórax anteroposterior portátil. Edema agudo de pulmón. Se observa infiltrado alveolointersticial difuso bilateral. Cardiomegalia II/IV. Prótesis mecánica en posición aórtica y mitral y suturas de esternotomía media

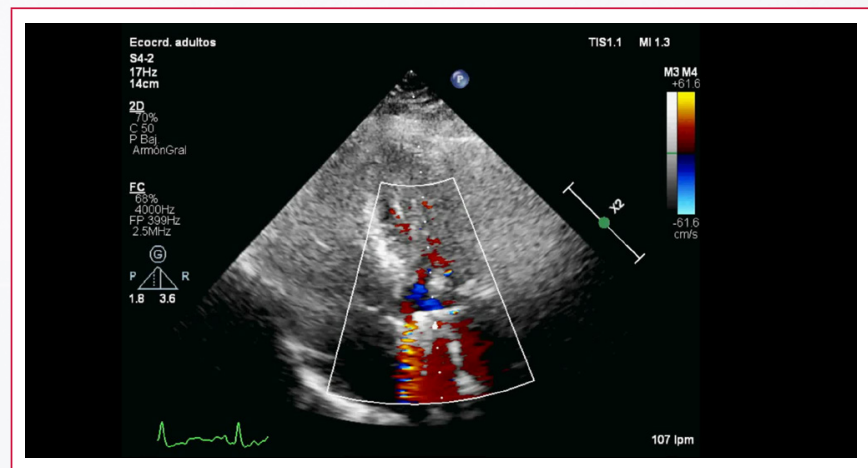

Vídeo 1. ETT, plano de 5 cámaras color. Sobre todo, es evidente la presencia de un flujo turbulento a través de la válvula mitral protésica. La frecuencia cardíaca durante la realización del estudio era de 107 lpm. Al ser un ecocardiograma realizado de forma emergente, no disponemos de más imágenes para mostrar

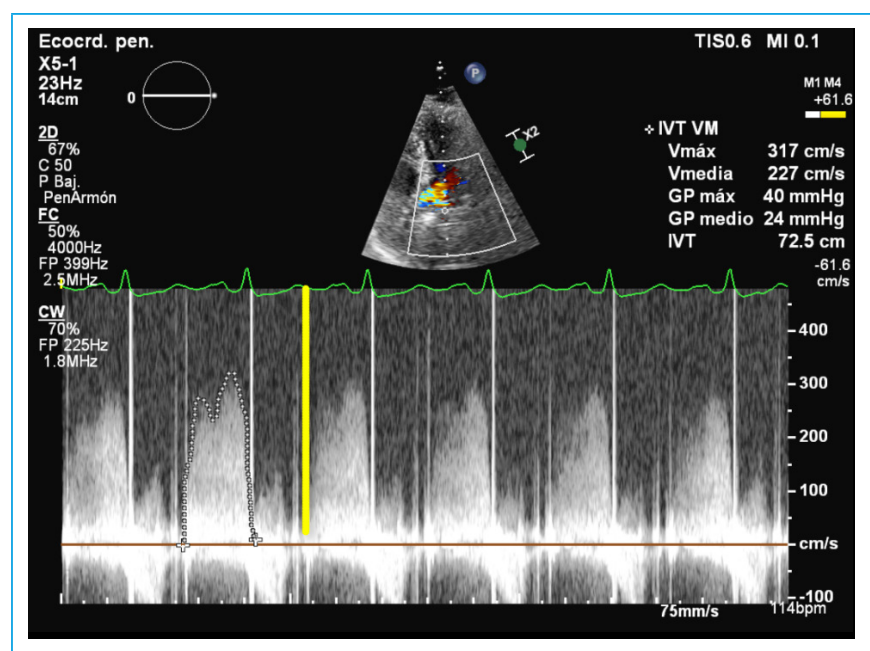

Figura 2. Doppler continuo mitral. Se observa un aumento marcado del gradiente transvalvular mitral. Asimismo, la línea de apertura valvular mitral no es densa, lo que sugiere que los discos de la válvula no se mueven bien. Hemos colocado una línea amarilla donde debería verse la línea de apertura mitral

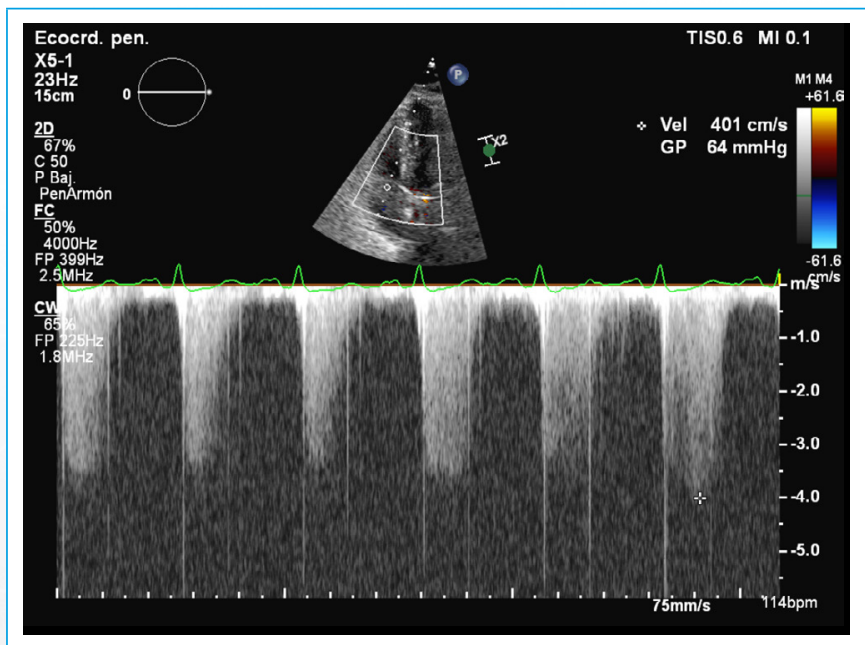

Figura 3. Doppler continuo tricúspide. Se observa que la paciente tiene un gradiente ventriculoauricular elevado $(64 \mathrm{mmHg})$, lo que permite estimar una presión arterial pulmonar sistólica elevada

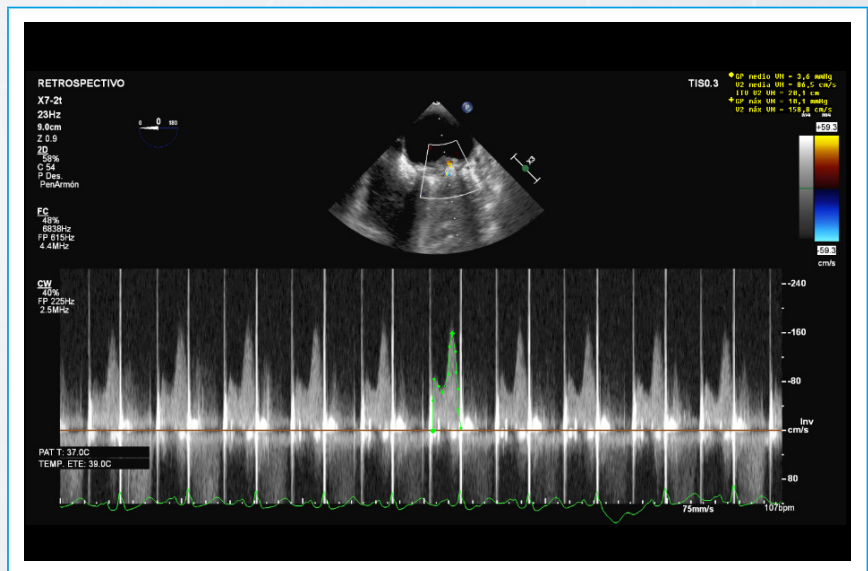

Figura 4. Doppler continuo mitral. Se observa una disminución marcada del gradiente transvalvular mitral, con respecto al ecocardiograma realizado antes de la administración de fibrinolítico. Asimismo, la línea de apertura valvular mitral es densa, lo que sugiere que los discos de la válvula se mueven (véase Vídeo 2) 


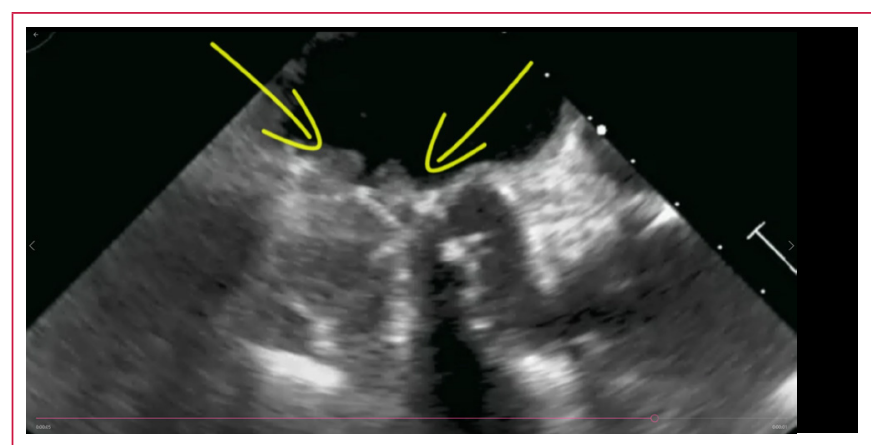

Vídeo 2. ETE, plano de tracto de salida del ventrículo izquierdo. Las flechas amarillas señalan la persistencia de trombos en ambos discos de la prótesis

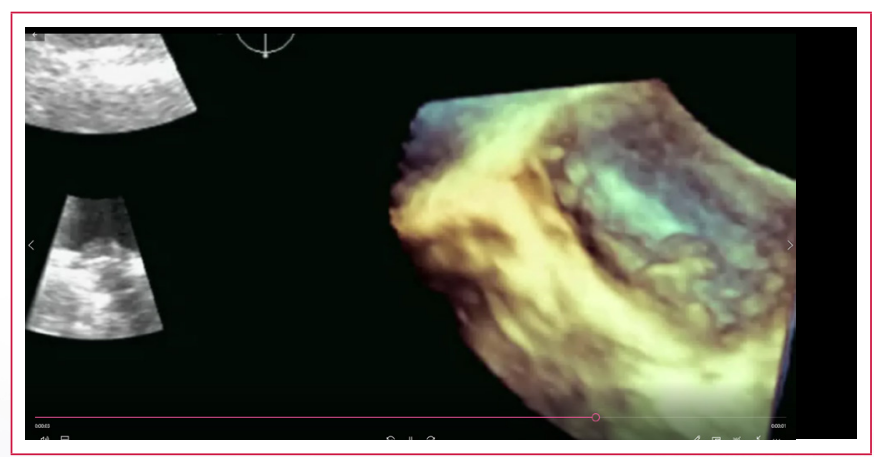

Vídeo 3. ETT, 3D. El cuadro que queda en pausa muestra los trombos sobre ambos discos en aurícula izquierda. El vídeo hace zoom sobre la zona de interés para que se puedan ver los trombos con mayor nitidez en pausa y en movimiento

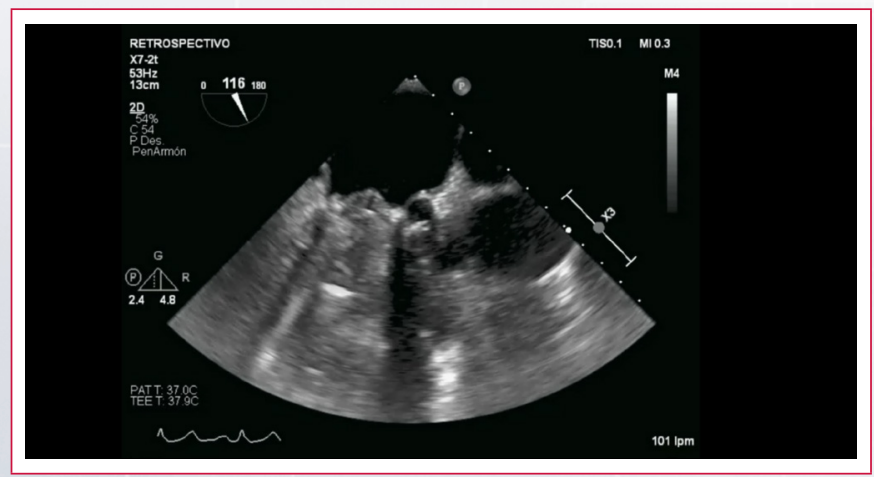

Vídeo 4. ETE, plano de tracto de salida del ventrículo izquierdo. Se puede observar cómo el trombo tapizaba los discos valvulares sin solución de continuidad

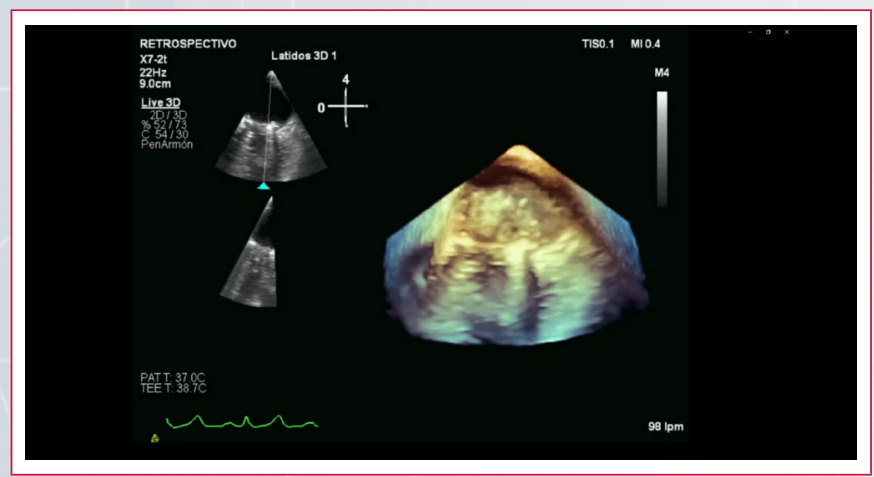

Vídeo 5. ETE, 3D. Se observa la progresión del tamaño del trombo valvular (círculo amarillo), que, además de producir un aumento del gradiente transvalvular, por sus características consideramos que tenía riesgo de embolización

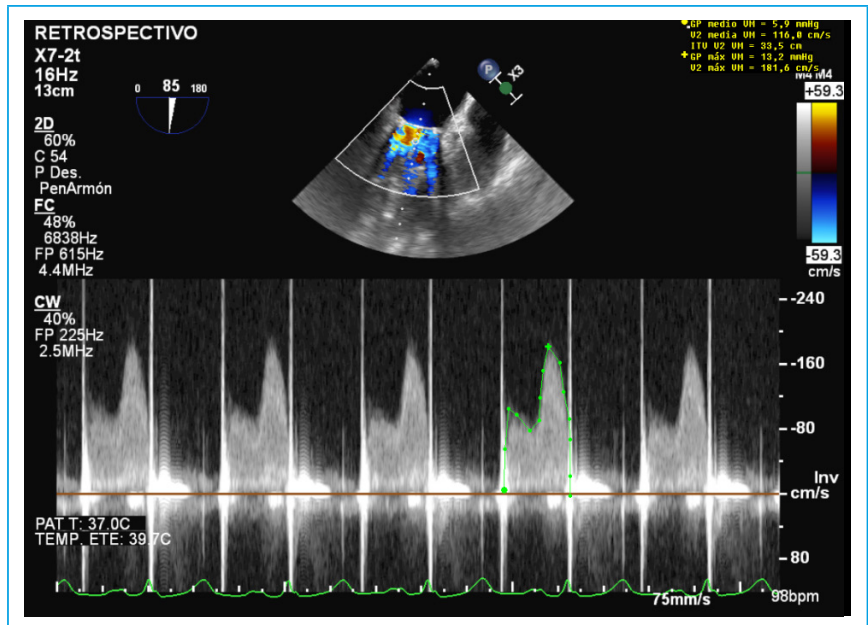

Figura 5. Doppler continuo mitral. Se observa un leve aumento del gradiente transvalvular mitral compatible con progresión de la extensión de la trombosis

\section{Discusión}

Existen cuatro mecanismos fundamentales por los que una prótesis valvular puede convertirse en disfuncionante (aunque en ocasiones pueden coexistir de forma conjunta):

- Trombosis protésica.

- Crecimiento de pannus (definido como una reacción biológica exagerada con proliferación de fibroblastos y matriz extracelular).

- Degeneración valvular.

- Endocarditis.

Ante el diagnóstico de disfunción protésica, muchas veces el reto está en diferenciar entre una trombosis de la prótesis o una degeneración con desarrollo de pannus. Existen algunos datos que pueden ser de ayuda en el diagnóstico diferencial (Tabla 1).

\begin{tabular}{|c|c|c|}
\hline & Trombosis protésica & Pannus \\
\hline Patogénesis & $\begin{array}{l}\text { - Agregación de plaquetas, } \\
\text { generación de trombina } \\
\text { y formación de trombo }\end{array}$ & $\begin{array}{l}\text { - Generación de trombina } \\
\text { y depósito de fibrina } \\
\text { - Proliferación de } \\
\text { fibroblastos, depósito } \\
\text { de colágeno y } \\
\text { neoangiogénesis } \\
\end{array}$ \\
\hline $\begin{array}{l}\text { Presentación } \\
\text { clínica }\end{array}$ & $\begin{array}{l}\text { - Semanas/meses tras el } \\
\text { implante de la prótesis. } \\
\text { - Clínica aguda o subaguda } \\
\text { - INR por debajo del rango }\end{array}$ & $\begin{array}{l}\text { - Meses/años tras el } \\
\text { implante de la prótesis } \\
\text { - Clínica progresiva }\end{array}$ \\
\hline Imagen & $\begin{array}{l}\text { - Masa de mayor tamaño } \\
\text { y densidad } \\
\text { - Localización en región } \\
\text { auricular de prótesis } \\
\text { mitrales y en región } \\
\text { aórtica de prótesis aórticas } \\
\text { - Gran reducción de la } \\
\text { movilidad de los velos/ } \\
\text { discos }\end{array}$ & $\begin{array}{l}\text { - Masas de menor tamaño } \\
\text { y menor densidad } \\
\text { - } \text { Localizadas en cara } \\
\text { ventricular de ambas } \\
\text { prótesis } \\
\text { - } \text { Menor reducción de } \\
\text { movilidad de los velos/ } \\
\text { discos }\end{array}$ \\
\hline
\end{tabular}

Tabla 1. Diagnóstico diferencial de trombosis protésica y pannus

La incidencia de trombosis protésica mecánica (TPM) sintomática varía desde el 0,3 hasta el 1,3\% por año, con altos porcentajes (6\%) en aquéllos con anti- 
coagulación infraterapéutica. En un reporte de TPM se encontró que la terapia inadecuada con anticoagulación fue responsable hasta del 70\% de los casos. Aunque los estudios sean escasos, los casos de TPM en posición mitral son hasta dos veces más frecuentes que en posición aórtica y la TPM en posición tricuspídea se ha estimado hasta 20 veces más frecuente que la de lado izquierdo ${ }^{(1)}$.

La sospecha diagnóstica de TPM debe darse ante una insuficiencia cardíaca aguda o fenómeno embolico de reciente aparición. La ecocardiografía es una herramienta muy útil y, aunque no existen criterios definidos para la TPM, debería sospecharse cuando el gradiente transvalvular medio se eleva más del $50 \%$ respecto al valor basal y no se explica por otros factores como un estado de alto flujo (anemia o sepsis) o mismatch. De no disponer de los gradientes basales en el posquirúrgico cercano, el hallazgo de un gradiente por encima del valor normal descrito para el tipo y tamaño de válvula (> 95\% IC) deberá hacernos sospechar ${ }^{(2)}$

La ETT debe ser la primera prueba que se realice, y una evaluación completa incluye el gradiente medio transvalvular para el diagnóstico de obstrucción valvular significativa, la movilidad de los hemidiscos, identificando la presencia y severidad de la regurgitación (transvalvular o paravalvular), la identificación de imágenes compatibles con trombo, su tamaño, su movilidad y, finalmente, la función de los ventrículos izquierdo y derecho. La ETE permite una mejor caracterización de las imágenes (vegetación, pannus) y tamaño de trombos ${ }^{(3)}$

La bibliografía establece que la terapia fibrinolítica obtiene mejores resultados en pacientes con alto riesgo quirúrgico, situación basal NYHA I-II, con un primer episodio de trombosis valvular, trombo pequeño $\left(<1 \mathrm{~cm}^{2}\right.$ o móvil $<5 \mathrm{~mm}$ ), en ausencia de edema agudo de pulmón y contraindicaciones absolutas para la fibrinólisis.

La ETE no sólo aporta información al diagnóstico, sino que también permite monitorizar la respuesta terapéutica, estableciéndose como objetivo una reducción de al menos un 75\% del tamaño del trombo, pudiéndose realizar varios ciclos hasta alcanzar la dosis máxima acumulativa del agente fibrinolítico(4).

Los estudios comparativos entre cirugía y fibrinólisis aún resultan controvertidos. Un metaanálisis incluyo siete estudios observacionales en 598 pacientes concluyendo que la mortalidad fue similar con ambas, la restauración de la función valvular no fue significativamente mayor con la cirugía, sin embargo la cirugía se asoció a menor número de fenómenos embólicos, sangrados mayores y recurrencia de trombosis ${ }^{(5)}$. En cambio, otro metaanálisis de 48 estudios con 2.302 pacientes indicó que el porcentaje de mortalidad en los tratados con cirugía fue significativamente mayor que en los tratados con fibrinólisis(6). La cirugía se asoció con menor porcentaje de fenómenos tromboembólicos, aunque el porcentaje de ictus y sangrados fueron similares.

Finalmente, la terapia antitrombótica a largo plazo, una vez resuelta la trombosis valvular, no está bien definida; se describe hasta un $6 \%$ de recurrencia en una media de seguimiento de 10 meses. La optimización de la anticoagulación, incrementando en casos seleccionados el rango de NR 3,5-4,5 y añadiendo AAS de acuerdo con el riesgo hemorrágico, se basará en la opinión de expertos.

\section{Conclusión}

La ecocardiografía es una herramienta fundamental tanto en el diagnóstico como en la elección del tratamiento, la evaluación de la respuesta y seguimiento del paciente.

La fibrinólisis lenta parece ser una estrategia válida y con buenos resultados, sin embargo, siempre debemos individualizar la estrategia a seguir de acuerdo con criterios clínicos y ecocardiográficos.

\section{Ideas para recordar}

- Una de las principales causas de trombosis de prótesis mecánicas valvulares son las terapias puentes a dosis infraterapéuticas por temor al sangrado.

- El riesgo de trombosis de válvulas protésicas es mayor en posiciones derechas, seguido de la mitral y finalmente aórtica.

- En todo paciente portador de prótesis mecánica valvular, con clínica de insuficiencia cardíaca o fenómeno embólico reciente, debe realizarse ecocardiografía, siendo más rentable el estudio transesofágico.

\section{Bibliografía}

1. Roudaut R, Serri K, Lafitte S. Thrombosis of prosthetic heart valves: diagnosis and therapeutic considerations. Heart 2007; 93: 137-142.

2. Zoghbi WA, Chambers JB, Dumesnil JG, et al. Recommendations for evaluation of prosthetic valves with echocardiography and doppler ultrasound: a report from the American Society of Echocardiography's Guidelines and Standards Committee and the Task Force on Prosthetic Valves, developed in conjunction with the American College of Cardiology Cardiovascular Imaging Committee, Cardiac Imaging Committee of the American Heart Association, the European Association of Echocardiography, a registered branch of the European Society of Cardiology, the Japanese Society of Echocardiography and the Canadian Society of Echocardiography, endorsed by the American College of Cardiology Foundation, American Heart Association, European Association of Echocardiography, a registered branch of the European Society of Cardiology, the Japanese Society of Echocardiography, and Canadian Society of Echocardiography. J Am Soc Echocardiogr 2009; 22 (9): 975-1014.

3. Nishimura RA, Otto CM, Bonow RO, et al. 2014 AHA/ACC guideline for the management of patients with valvular heart disease: a report of the American College of Cardiology/American Heart Association Task Force on Practice Guidelines. J Am Coll Cardiol 2014; 63: 2438-2488.

4. Mutuberría-Urdaniz M, Rodríguez-Palomares JF, Ferreira I, et al. Non-obstructive prosthetic heart valve thrombosis (NOPVT). Really a benign entity? Int J Cardiol 2015; 197: 16-22.

5. Karthikeyan G, Senguttuvan NB, Joseph J, et al. Urgent surgery compared with fibrinolytic therapy for the treatment of left-sided prosthetic heart valve thrombosis: a systematic review and meta-analysis of observational studies. Eur Heart J 2013; 34: 1557-1566.

6. Castilho FM, De Sousa MR, Mendonça AL, et al. Thrombolytic therapy or surgery for valve prosthesis thrombosis: systematic review and meta-analysis. J Thromb Haemost 2014; 12: 1218-1228. 